\title{
Competencia mediática de jóvenes universitarios de Ecuador y Colombia
}

\section{Media Competences of Young University Students from Ecuador and Colombia}

\author{
Isidro Marín-Gutiérrez \\ Diana Rivera-Rogel \\ Universidad Técnica Particular de Loja (Ecuador) \\ Damian Mendoza-Zambrano \\ Universidad Laica Eloy Alfaro de Manabí (Ecuador) \\ Universidad de Quebec en Trois-Rivières (Canadá) \\ Ligia Inés Zuluaga-Arias \\ Universidad Católica Luis Amigó (Colombia)
}

El presente estudio muestra resultados de dos países latinoamericanos: Ecuador y Colombia. El objetivo general es conocer el tratamiento que asumen los jóvenes frente a la tecnología digital y los niveles de competencia mediática. Como objetivos específicos se plantea: definir los indicadores de referencia para medir el grado de competencia mediática en los jóvenes universitarios; evaluar de manera exploratoria los niveles y el grado de competencia mediática en las muestras seleccionadas de los dos países. La metodología utilizada para evaluar el grado de competencia mediática de los jóvenes de Ecuador y Colombia es la práctica de una aproximación exploratoria de corte descriptivo. Entre los hallazgos se evidencia la utilización de navegación a internet para habilidades más técnicas
The present study shows results from two Latin American countries: Ecuador and Colombia. The general objective is to find out how young people get by in the face of digital technology and levels of media competence. The specific objectives are to define the reference indicators to measure the degree of media competence of university students, and to evaluate, in an exploratory way, the levels and the degree of media competence in selected samples from the two countries. The methodology used to evaluate the degree of media competence of young people in Ecuador and Colombia is to carry out an exploratory approach of a descriptive nature. Among the findings it is worth noting the use of Internet browsing for more technical and operational skills than 
y operativas que valorativas al nivel de la competencia mediática. Se detecta la necesidad de establecer procesos de alfabetización mediática sistémicos y continuos. El análisis de estos datos podría ser considerado como base para el establecimiento de políticas públicas en educación mediática. Esta temática en estos países es incipiente y se agota en líneas generalistas propuestas en documentos públicos, concerniente a los planes de educación e iniciativas particulares que en la actualidad se muestran desarticuladas.

Palabras clave: competencias mediáticas, estrategia educomunicativa, estudiantes universitarios, contextos múltiples, alfabetización digital. valuations at the level of media competence. The need to establish systematic and continuous media literacy processes is identified. The analysis of these data could be considered a basis for the establishment of public policies in media education. This issue in these countries is emerging and it is exhausted in general lines as proposed in public documents, concerning the education plans and particular initiatives that are currently fragmented.

Key words: media competences, educommunicative strategy, university students, multiple contexts, digital literacy.

$\mathrm{L}$ os medios de comunicación son parte del vivir diario de la humanidad en las sociedades actuales. Sin ellos no es posible una interacción que permita una comunicación entre generaciones en la vida social (Clarke, 2020). Los medios de comunicación nos transmiten formas de actuar y nos influencian en cómo valorar las costumbres y tendencias (Sánchez-Carrero y Aguaded-Gómez, 2013).

Desde las primeras relaciones en la escuela con maestros y compañeros de estudio, los procesos de mediación social se generaban a través del diálogo y la escritura como elemento de soporte para adquirir habilidades para leer, comprender y actuar a partir de los códigos escritos aprendidos en el proceso de formación escolar (Cabero-Almenara y Valencia-Ortiz, 2019). En la actualidad, los soportes digitales son los nuevos intermediarios tecnológicos que forman parte de nuestras realidades sociales en los escenarios escolares (Drotner, 2019). Esto ha permitido la aparición de conceptos como alfabetización mediática, competencias mediáticas y competencias ciudadanas, términos que han generado reflexiones en torno a las nuevas formas de leer estos fenómenos educativos (Tirado-Morueta et al., 2017).

Así pues, es necesaria una educación en medios que los ayude a construir su identidad, también a reconocer el mundo con una mirada crítica y con la capacidad creativa de interpretarlo de forma libre y expresiva. Los medios de comunicación transmiten valores ideológicos. Los medios construyen e interpretan la realidad social (Fontcuberta, 1997). 
La educación mediática inicia la crisis de quién debe asumirla. El sistema escolar no termina por articularla y en el contexto social parece prevalecer la creencia de que la sola interacción con estos artefactos tecnológicos nos faculta para hacerle frente a sus impactos (Jitjumnong, Chujai y Kamata, 2020). Sin embargo, la interacción con estos dispositivos culturales estratégicos ha permitido generar espacios de reflexión que deben asumir los escenarios educativos como la escuela, desde las implicaciones expresivas, técnicas, culturales y axiológicas, al igual que organismos internacionales (Juan, Yasmín y Milton, 2020). Desde 1964, la UNESCO está promoviendo encuentros internacionales que analicen problemáticas y nuevas estrategias para la educomunicación (Alcolea-Díaz, Reig y Mancinas-Chávez, 2020).

En el contexto actual sería difícil imaginar el devenir social y cultural de la humanidad sin la interacción que se realizan diariamente con los diferentes medios de comunicación. Los medios de comunicación nos ayudan a ver más allá de nuestros horizontes, activan nuestra curiosidad y fomentan nuestra imaginación. Sin ellos nos sentimos vacíos. Con la televisión, la radio o nuestro smartphone vencemos a la soledad (García-Leguizamón, 2010: 281), y más en estos momentos en los que el COVID-19 nos ha obligado a encerrarnos en nuestras casas desconociendo cuándo podremos volver a salir.

En este siglo debe decidirse cuáles son los caminos más asertivos para educar con, en y frente a los medios. Las razones para hacerlo siempre han existido, pero hoy se agudizan por la infoxicación (o sobrecarga informativa), el aumento de aparatos tecnológicos en nuestros hogares (muchos de ellos de carácter de inteligente como lavadoras, batidoras, frigoríficos, televisiones o altavoces), las modas o la obsolescencia vertiginosa en donde una computadora queda obsoleta en dos años. Vivimos tiempos extraños que nos hacen inmovilizar nuestra capacidad de reacción, "provocando una fe ciega y sin cuestionamientos" (Sánchez-Carrero y Contreras-Pulido, 2012: 25).

Detrás de la expresión "competencia mediática" hay discusiones crítico-reflexivas e instrumentales, todas conducentes al desarrollo sistémico de habilidades desde diferentes dimensiones que ayuden a las personas a interactuar en una realidad creada por los medios (García-Leguizamón, 2010).

Para generar transformaciones, la apropiación de este universo tecnológico requiere de un sustrato: sin competencias y alfabetización mediática ¿cómo afrontar estos consumos culturales? Hay que desarrollar habilidades cognitivas para interactuar con estos bienes culturales, aprender a discernir "y seleccionar entre una gama muy amplia de oferta formativa e informativa" (Huergo y Trenado, 2010: 75). Y para lograrlo se requiere de un proyecto de alfabetización mediática que genere competencias mediáticas. Una alfabetización mediática que sea crítica y a la vez liberadora. No necesitamos una formación como usuarios de un nuevo dispositivo tecnológico (Rivera et al., 2017).

No se trata de instrumentalización, sino del desarrollo de altas capacidades metacognitivas exigidas para afrontar las demandas complejas del ecosistema mediático (Milenkova, Keranova y Peicheva, 2020), es decir, la capacidad de preguntarse los porqués de este ecosistema. Las competencias mediáticas se constituyen en una combinación de habilidades cognitivas y prácticas interrelacionadas, 
conocimientos, valores, actitudes, emociones y otros componentes sociales que se movilizan a la vez para llevar a cabo una acción efectiva (Guex y Stiefel, 2010: 32).

Podría afirmarse que una alfabetización mediática asertiva debe generar competencias mediáticas adecuadas; de ahí la importancia de este último concepto en los abordajes actuales de la educación en medios. Si puede determinarse el nivel de competencia de quienes han participado o no en alfabetizaciones mediáticas, es posible fortalecer los procesos educativos mediáticos o saber cuáles son las rutas a seguir para hacerle frente a esta necesidad formativa.

Lograr este tipo de formación implica considerar las dimensiones que debe suponer esta competencia - lenguaje, tecnología, procesos de interacción, procesos de producción y difusión, ideología y valores y estética-, cada una de ellas diseñada en dos aspectos: el de la creación de mensajes de cada uno y el de la interacción con mensajes de otros (Ferrés y Piscitelli, 2012: 75).

Pensar en competencias mediáticas en países como Colombia y Ecuador, donde el abordaje de la relación medios-educación difícilmente ha superado la fase tecnologicista de la educación mediática, es doblemente retador. Las investigaciones que presentan este manuscrito se nutren de la experiencia española de más de una década en el abordaje de las competencias mediáticas y de casi cincuenta años en la articulación del tema medios con lo educativo y lo sociocultural (Scolari, 2016).

Estas investigaciones se adaptan de la memoria técnica del Proyecto que cerró en el 2014: I+D, la competencia en comunicación audiovisual en un entorno digital. Se trata de un diagnóstico de necesidades en tres ámbitos sociales: el de los profesionales de la comunicación, , el de la enseñanza obligatoria y el de la enseñanza universitaria. En el estudio participaron la Universitat Pompeu Fabra, la Universidad de Huelva y la Universidad de Valladolid. Y en la actualidad es replicado desde diferentes perspectivas entre las universidades que forman parte de la Red Interuniversitaria Euroamericana de Investigación sobre Competencias Mediáticas para la ciudadanía, que tiene a más de 60 investigadores de 14 países europeos y latinoamericanos: Argentina, Brasil, Bolivia, Chile, Colombia, Cuba, Ecuador, España, Italia, México, Perú, Portugal y Venezuela $(<$ https://redalfamed. $\operatorname{org} />$ ).

El objetivo general es conocer el comportamiento que los jóvenes tienen frente a las tecnologías y cuál es el nivel de competencia mediática que poseen desde el acercamiento exploratorio de corte descriptivo para la evaluación del grado de competencia mediática en los jóvenes de los dos países. Y los objetivos específicos: definir los indicadores de referencia para medir el grado de competencia mediática en los jóvenes universitarios; evaluar de manera exploratoria los niveles y el grado de competencia mediática en las muestras seleccionadas de los dos países y detectar algunas necesidades y carencias de los ámbitos sociales específicos en relación con los niveles de competencia mediática desde los estudios de Ferrés (2007) y Ferrés y Piscitelli (2012).

En tal virtud, en este estudio se presentan los resultados cuantitativos de los dos países, hallazgos que hacen inminente la necesidad de establecer procesos de alfabetización mediática sistémicos y continuos. El análisis de estos datos podría 
ser considerado como base para el establecimiento de políticas públicas en educación mediática, temática que en estos países es incipiente y se agota en algunas líneas generalistas propuestas en documentos públicos sobre los planes de educación o en iniciativas particulares desarticuladas.

La promoción de las competencias mediáticas no puede asociarse solo con los espacios educativos formalizados, ni relacionarse con procesos pedagógicos y didácticos tradicionalistas. Este proceso formativo es un reto social que involucra a diferentes actores y que impacta el ejercicio de la ciudadanía, máxime, cuando en la actualidad participamos de contextos globalizados e hipercomunicados (Gozálvez-Pérez y Contreras-Pulido, 2014). Esto significa que ser ciudadano hoy es ser ciudadano en lo mediado y para ello se requiere de la aprehensión de conocimientos, habilidades y actitudes para que la ciudadanía en democracia haga valer su protagonismo en el terreno político, jurídico, social, económico, ecológico o intercultural, para no caer en nuevas formas de sumisión en tales ámbitos (Gozálvez-Pérez y Contreras-Pulido, 2014).

Si quieren fortalecerse estas iniciativas, es necesario partir de evaluaciones reales de la competencia mediática de los jóvenes de estos países como uno de los colectivos más importantes. Estos estudios y la difusión internacional de sus resultados resultan esenciales porque son una muestra de la situación en educación mediática de países latinoamericanos que necesitan fortalecer sus procesos ciudadanos y políticos, y que para lograr estos propósitos requieren el concurso de formar a una juventud en, con y ante los medios en los escenarios escolares para educar a una ciudadanía comprometida con el devenir social y político.

\section{MARCO TEÓRICO}

El aprendizaje desde que nacemos hasta que morimos es un hecho irrevocable en la sociedad de las pantallas (Caldeiro-Pedreira y Aguaded-Gómez, 2015). La educomunicación va a ser parte de la formación de las personas permanente y necesaria por distintas razones tanto personales como profesionales allá donde tengamos que interactuar con aplicaciones digitales.

Con los cambios tecnológicos de los años noventa del siglo pasado hemos comenzado a hablar sobre la educomunicación aplicada a las TIC (Tecnologías de la Información y Comunicación). La aparición del término alfabetización digital se ha ido dirigido a dos aspectos principales: el primero interesado principalmente en el software o en la herramienta digital y el segundo interesado en aspectos de utilización de la herramienta para aspectos participativos, sociales o comunicacionales (Martínez et al., 2013).

Es indiscutible que las TIC han entrado a formar parte de nuestras vidas de manera acelerada y han ocasionado consecuencias dentro del ámbito tecnológico. Pensamos que el ritmo de crecimiento y de innovación seguirá aumentando. La alfabetización es un concepto que ha variado a lo largo de la historia. La alfabetización digital va a comprender nuevas formas que se tienen que incorporar a la institución educativa. La alfabetización digital va a comprender competencias en producción, en lenguaje audiovisual, en recursos informáticos, en habilidades 
de buscar la información en las redes, en selección de información y de transformación para unos fines indicados (Moreira, 2014). La alfabetización digital va a permitir que una persona realice habilidades en ambientes digitales a través de la hipermedia con textos, sonidos, imágenes y videos digitales, y que aprenda a trabajar en comunidad aplicando los nuevos conocimientos que ha adquirido (Gros-Salvat y Contreras, 2006).

En el mundo digital se hace necesario alcanzar habilidades mínimas para aprovechar la innovación tecnológica que nos rodea, con el fin de conocer parámetros y herramientas nuevas que nos ayuden en el crecimiento diario detrás de este auge que está marcando a las nuevas enseñanzas. Incluso está unida con la red para trabajar en un entorno digital. Los cambios de la tecnología son constantes en el mundo tradicional y dejan que usuarios frecuentes de esta herramienta generen nuevas interpretaciones tecnológicas; innovar a través de la tecnología hace que los usuarios sean partícipes de nuevos escenarios formativos.

La alfabetización no es solo un tema de lectoescritura y matemáticas básicas, sino también un aprendizaje profundo sobre el entorno que tiene que ayudar al individuo a estar cómodo en su realidad y a poder actuar de manera reflexiva. Gracias a ello se deben adquirir todos los recursos que ofrece la sociedad para poder interactuar de una manera crítica con la cultura existente. La alfabetización es una necesidad del ciudadano ante la sociedad que le toca vivir (Moreira, 2014).

La alfabetización digital es aquella práctica que permite al usuario tener bases para organizar, entender y analizar información real-verídica por medio de la tecnología. Este cambio se demuestra por diferentes razones: en primer lugar, por la llegada del mundo virtual donde con un click localiza todo lo que busca; en segundo lugar, es importante la alfabetización en nuestro aprendizaje con las tecnologías porque así podemos comprender la importancia de nuestra rutina diaria, con ellas nos podemos especializar en diferentes ramas de estudio permitiendo el libre acceso a cursos online, con el único objetivo de acatar conocimientos.

La sociedad de las pantallas crea nuevas formas de alfabetización digital para la formación ciudadana en competencias digitales. Para la nueva sociedad son fundamentales nociones en comunicación digital o la participación en redes (Gros-Salvat y Contreras, 2006). La alfabetización digital, desde su misma definición y contenidos, es materia de discusión. Inicialmente, el concepto de alfabetización lectora fue entendido como la simple capacidad de leer y escribir. Tal concepción fue ampliada por la UNESCO (1986) a través del concepto de alfabetización funcional, describiendo a las personas alfabetizadas cuando pueden leer, escribir y comprender lo que han escrito o leído. La alfabetización funcional se refiere a cuando una persona puede realizar todas las actividades necesarias para el funcionamiento eficaz de su comunidad (Bravo y Contreras, 2000: 37). Por ejemplo, las actividades del profesorado están cambiando gracias a las TIC. Está cambiando de metodología para tener nuevos objetivos educativos. El profesorado ya no es un mero transmisor del conocimiento, sino un facilitador de los procesos de enseñanza y de aprendizaje. Las TIC van a ser fundamentales tanto en la práctica educativa como en las actividades creadas por el profesorado. El alumnado cuenta con habilidades en el uso de las TIC por su contacto desde la 
niñez. Aprenden de manera autónoma y adquieren así capacidades y habilidades que los acercan hacia sus propias metas.

Es necesario también analizar que en algunos contextos latinoamericanos las variables sociodemográficas tienen influencia en el acceso a los materiales, así como el aprovechamiento académico o el conocimiento del lenguaje (Mendoza et al., 2017: 520).

Las habilidades del ciudadano en la sociedad de las pantallas necesitan de la ayuda de la tecnología. Las TIC ofrecen nuevas posibilidades al mundo educativo (ofrecen nuevas formas de educación, de aplicar lo aprendido, lo importante ya no son los contenidos, sino la interacción que se produzca con ellos, el acceso a la información, etc.). Gracias a las TIC, los alumnos van a tener un aprendizaje activo y colaborativo. El docente debe incorporarlo a su quehacer diario. La alfabetización digital no es ni más ni menos que la formación para vivir en su nuevo entorno y debe adquirirse en el hogar y en las instituciones educativas (Moreiro et al., 2010).

\section{METODOLOGÍA}

\section{MATERIAL Y MÉTODOS}

El objetivo principal de este estudio es saber cuáles son las diferencias desde las seis dimensiones definidas por Ferrés (2007) y Ferrés-i Prats y Piscitelli (2012) en estudiantes universitarios (17-22 años). En este artículo determinaremos el nivel de competencia audiovisual de las seis dimensiones: 1) Lenguaje; 2) Tecnología; 3) Procesos de interacción; 4) Procesos de producción y difusión; 5) Ideología y Valores y 6) Estética. La hipótesis de partida para este estudio es que existen diferencias en cuanto a las dimensiones.

\section{Metodología}

Según los datos de este estudio, existen experiencias previas sistemáticas y prolongadas de evaluación sobre el nivel de adquisición en competencias mediáticas en alumnado universitario (Sari et al., 2020). En este caso, nuestra investigación es precursora de la exploración de las competencias mediáticas en el ámbito universitario entre alumnado universitario en Ecuador y Colombia.

La metodología que se utilizó en esta investigación fue cuantitativa con el uso del cuestionario digital. El instrumento básico fue la encuesta online. Para diseñar el cuestionario hemos seguido el formato para medir las competencias mediáticas de Ferrés-i Prats y Piscitelli (2012). ¿Por qué hemos utilizado este formato y no otro? Básicamente porque es un modelo ya validado, muy utilizado y citado por otros investigadores como figura en el gráfico $\mathrm{n}^{\mathbf{0}} 1$ elaborado con las citas en la base de datos de Scopus de Ferrés-i Prats y Piscitelli (2012), siendo utilizado por Izquierdo-Iranzo y Gallardo-Echenique (2020) o Pérez-Rodríguez et al. (2019), entre otros. 
Gráfico 1. Número de citaciones por año del artículo de Ferrés-i Prats y Piscitelli (2012) en la base de datos de Scopus

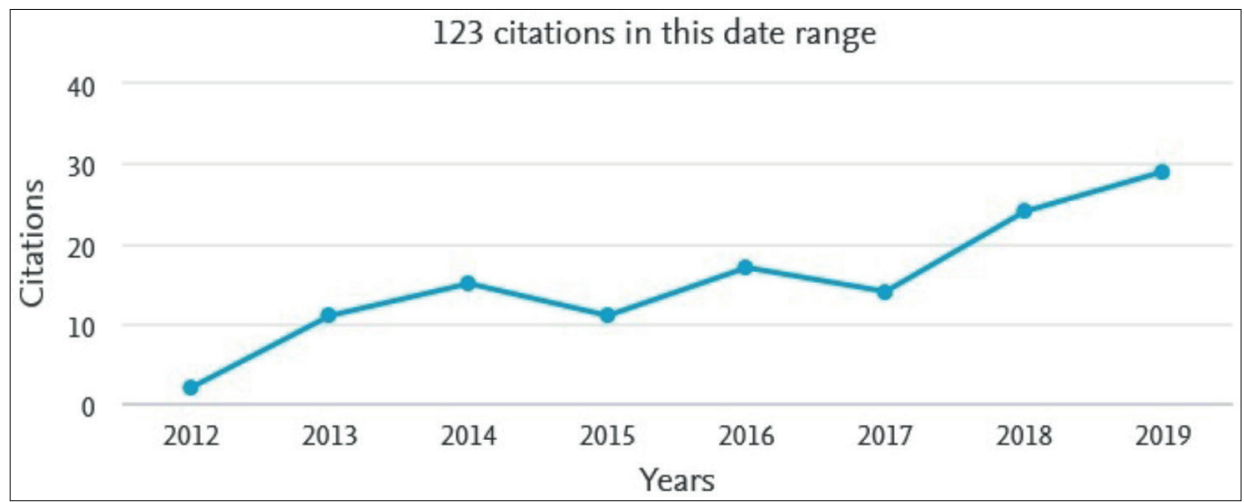

Fuente: SCOPUS <https://bit.ly/2WNJCYj>.

Una vez terminado el cuestionario por un equipo multidisciplinar de profesores internacionales, se realizó una prueba piloto sobre una muestra de 60 individuos de varias instituciones de los dos países que conforman el estudio. No hubo ningún tipo de dificultad a la hora de responder al cuestionario. Estas muestras de los pretest fueron sometidas a análisis de fiabilidad y de consistencia interna con el índice Alfa de Crombach, y alcanzaron valores de 0,83. Consideramos que el resultado es fiable. No se realizaron cambios a la versión postest del cuestionario. Los análisis de fiabilidad y los juicios de los expertos internacionales fueron el proceso de validación del cuestionario. El cuestionario consta de 23 preguntas.

El cuestionario se realizó durante el año 2017 (concretamente desde el 8/01/2017 hasta el 1/07/2017) en Colombia a 484 estudiantes y en Ecuador a 1.616 estudiantes, de acuerdo a distribución. Las encuestas a universitarios se pueden consultar en <https://goo.gl/XFwgP3>. Se recogió un total de 2.100 cuestionarios en los dos países. El cuestionario contenía preguntas que atendían a las seis dimensiones propuestas por Ferrés (2007) en la tabla 1.

Tabla 1. Preguntas por dimensiones y cuestionarios

\begin{tabular}{l|l} 
& Cuestionario a universitarios \\
\hline Lenguaje & Preguntas 13,14 y 16 \\
\hline Tecnologías & Preguntas $8,10,11$ y 12 \\
\hline Interacción & Preguntas 9, 17, 18, 19 y 20 \\
\hline Producción y Difusión & Preguntas 22 y 23 \\
\hline Ideología y Valores & Preguntas 21 \\
\hline Estética & Preguntas 15 \\
\hline \multicolumn{2}{l}{ Fuente: elaboración propia. }
\end{tabular}


El diseño fue descriptivo, ya que nuestra investigación se centró en analizar cuál era el nivel de las variables en un determinado momento para describir los fenómenos y estudiar su incidencia (Hernández-Sampieri, Fernández-Collado y Baptista-Lucio, 2014). Otro paso fue descargar las bases de datos creadas por el sistema y limpiarlo de posibles errores. Más tarde se recategorizaron todas las respuestas con el programa estadístico SPSS (versión 23), con la rúbrica de evaluación creada durante el diseño de las encuestas para medir las diferentes dimensiones sobre competencias mediáticas. Los indicadores presentados son genéricos y flexibles, adaptados a la situación educativa.

Tras obtener los datos básicos, se realizaron análisis estadísticos empleando el nivel de significancia del 95\%, así como las pruebas de Chi-cuadrado $\left(\chi^{2}\right)$ para diferenciar si las frecuencias observadas en cada una de las variables categóricas presentan frecuencias que se pueden encontrar si las muestras fueron extraídas de una población con una específica distribución.

\section{Población y MUESTRA}

Nuestra investigación tiene una población estudiantil universitaria de dos países. La muestra es de estudiantes universitarios de 17-22 años. El universo de estudio fueron alumnos universitarios tanto de centros públicos como privados. Se aplicó un total de 2.100 cuestionarios en estudiantes universitarios. La muestra fue seleccionada aleatoriamente por universidades de ambos países. Las variables independientes son la edad, el sexo y la universidad a la que pertenecen. Queda por adelantado que con esta muestra no pretendemos una significancia poblacional.

\section{RESULTADOS}

\section{LENGUAJE}

El 47,7\% de los colombianos se encuentra altamente de acuerdo con respecto a los mensajes de los medios, y el 40,4\% de los ecuatorianos, también. Existe una diferencia significativa del $15,5 \%$ entre los colombianos y los ecuatorianos en el grado alto de acuerdo (véase tabla 2). 
Tabla 2. Resultados de la dimensión de Lenguaje

\begin{tabular}{l|l|l|l|l|l|l} 
Lenguaje & \multicolumn{2}{l}{ Ecuador } & \multicolumn{2}{l}{ Colombia } \\
\hline Pregunta & Baja & Media & Alta & Baja & Media & Alta \\
\hline $\begin{array}{l}\text { 13. Respecto a los mensajes de los medios, } \\
\text { indica tu grado de acuerdo con estas } \\
\text { afirmaciones. }\end{array}$ & $12,9 \%$ & $46,6 \%$ & $40,4 \%$ & $9,6 \%$ & $42,8 \%$ & $47,7 \%$ \\
\hline $\begin{array}{l}\text { 14. Indica tu grado de acuerdo con el } \\
\text { significado de los diferentes lenguajes en } \\
\text { este fragmento de la película. }\end{array}$ & $15 \%$ & $37,7 \%$ & $48,4 \%$ & $15,4 \%$ & $31,7 \%$ & $52,9 \%$ \\
\hline $\begin{array}{l}16 . \text { Indica tu grado de acuerdo con la } \\
\text { importancia de estos elementos artísticos en } \\
\text { el significado de este videoclip. }\end{array}$ & $20,7 \%$ & $34,6 \%$ & $44,8 \%$ & $18,9 \%$ & $28,5 \%$ & $52,6 \%$ \\
\hline
\end{tabular}

Fuente: elaboración propia.

Se observa que el 52,9\% de los colombianos se encuentra con la mayor participación en el grado alto de acuerdo con el significado de los diferentes lenguajes (verbal, visual y musical) en el fragmento de la película y el 48,4\% los ecuatorianos, también. El 52,6\% de los colombianos presenta un grado alto de acuerdo con la importancia de elementos artísticos en el significado del videoclip, respecto al $44,8 \%$ de ecuatorianos.

Se valida la hipótesis nula, Ho, que plantea la igualdad de las medias proporcionales de los dos países en los diferentes factores (Canavos, 1988). Es decir:

$$
\begin{aligned}
& H_{o}: \Pi_{\text {Colombia }}=\Pi_{\text {Ecuador }} \\
& H_{1}: \Pi_{\text {Colombia }} \neq \Pi_{\text {Ecuador }}
\end{aligned}
$$

Donde:

$\Pi_{\text {Colombia }}=$ Proporción de individuos que cumplen con la característica objeto de estudio en Colombia.

$\Pi_{\text {Ecuador }}=$ Proporción de individuos que cumplen con la característica objeto de estudio en Ecuador.

El test de prueba será:

$$
Z_{p_{C}-p_{E}}=\frac{p_{\text {Colombia }}-p_{\text {Ecuador }}}{\sqrt{\frac{p_{C}\left(1-p_{C}\right)}{n_{C}}}+\frac{p_{E}\left(1-p_{E}\right)}{n_{E}}}
$$

Luego se procede a realizar la regla de decisión con un nivel de significancia del ,05:

Así:

Si $Z_{p_{c}-p_{E}} \geq Z \alpha / 2$ 
Es decir:

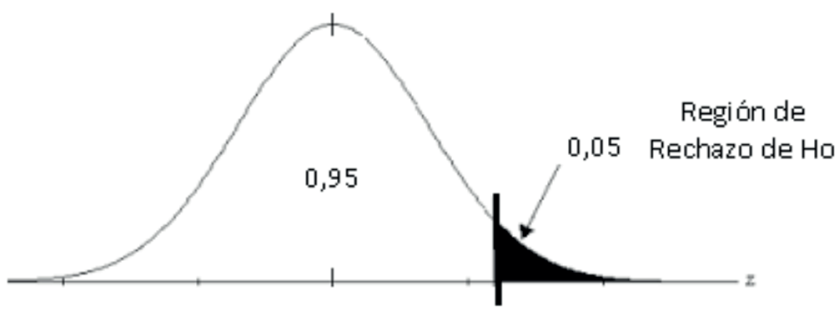

1,96

Con una confianza del 95\% se puede concluir que existe una diferencia estadísticamente significativa entre Colombia y Ecuador con respecto a los mensajes de los medios y el grado de importancia de estos elementos artísticos en el significado del videoclip visualizado (véase tabla 3).

Tabla 3. Diferencia estadística de medias proporcionales

\begin{tabular}{l|l|l|l|l|l} 
& \multicolumn{2}{|c|}{ Proporción Pi } & $\begin{array}{l}\text { Estadístico } \\
\text { de prueba }\end{array}$ & $\begin{array}{l}\text { Valor } \\
\text { crítico } \\
\text { Z } \alpha / 2\end{array}$ & $\begin{array}{l}\text { Regla de } \\
\text { decisión }\end{array}$ \\
\hline Pregunta & Colombia & Ecuador & & 1,96 & $\begin{array}{l}\text { Se rechaza } \\
\text { Ho }\end{array}$ \\
\hline $\begin{array}{l}\text { 13. Respecto a los mensajes de los medios, } \\
\text { indica tu grado de acuerdo con estas } \\
\text { afirmaciones. }\end{array}$ & $47,7 \%$ & $40,4 \%$ & 2,832 & 1,96 & $\begin{array}{l}\text { Se acepta } \\
\text { Ho }\end{array}$ \\
\hline $\begin{array}{l}\text { 14. Los diferentes lenguajes en este } \\
\text { fragmento de película. }\end{array}$ & $52,9 \%$ & $48,4 \%$ & 1,739 & 1,96 & $\begin{array}{l}\text { Se rechaza } \\
\text { Ho }\end{array}$ \\
\hline $\begin{array}{l}\text { 16. De estos elementos artísticos en el } \\
\text { significado del videoclip. }\end{array}$ & $52,6 \%$ & $44,8 \%$ & 3,018 & &
\end{tabular}

Fuente: elaboración propia.

En el factor del significado de los diferentes lenguajes (verbal, visual y musical) en el fragmento de la película no existe diferencia significativa estadística con un nivel 5\%.

\section{TeCNOLOGíA}

El 43,6\% de los colombianos opina que se encuentran con un alto grado de conocimiento de los recursos y el 37,6\% los ecuatorianos, también. Se observa que los dos países, Colombia y Ecuador, presentan similitudes en cuanto al navegador que más utiliza; es decir, el 27,3\% de los colombianos tiene un alto grado de uso del navegador y el 26,3\% de los ecuatorianos, también. El 56\% de los colombianos tiene un alto grado de razón en por qué utilizan el navegador y el 46,1\% de los ecuatorianos presenta altas razones de uso. El 35,94\% de los colombianos muestra un bajo grado en utilizar los pasos para realizar una búsqueda en internet y el 30,4\% refleja que los ecuatorianos hacen un bajo uso de los pasos para realizar la búsqueda. 
Tabla 4. Resultados de la dimensión de Tecnología

\begin{tabular}{|c|c|c|c|c|c|c|}
\hline \multirow{2}{*}{$\begin{array}{l}\text { Dimensión de Tecnologías } \\
\text { Pregunta }\end{array}$} & \multicolumn{3}{|c|}{ Ecuador } & \multicolumn{3}{|c|}{ Colombia } \\
\hline & Baja & Media & Alta & Baja & Media & Alta \\
\hline $\begin{array}{l}\text { 8. Indica el grado de conocimiento que } \\
\text { tienes de los siguientes recursos. }\end{array}$ & $29,9 \%$ & $32,5 \%$ & $40,4 \%$ & $29,4 \%$ & $42,8 \%$ & $47,7 \%$ \\
\hline 10. ¿Cuál es el navegador que más utilizas? & $55 \%$ & $37,7 \%$ & $18,7 \%$ & $62,1 \%$ & $31,7 \%$ & $52,9 \%$ \\
\hline $\begin{array}{l}\text { 11. Señala la razón principal por la que lo } \\
\text { utilizas. }\end{array}$ & $19,8 \%$ & $34,6 \%$ & $34,1 \%$ & $17,4 \%$ & $28,5 \%$ & $52,6 \%$ \\
\hline $\begin{array}{l}\text { 12. ¿Qué pasos sigues para realizar una } \\
\text { búsqueda en internet? }\end{array}$ & $28,3 \%$ & $41,3 \%$ & $30,4 \%$ & $30,5 \%$ & $33,6 \%$ & $35,9 \%$ \\
\hline
\end{tabular}

Fuente: elaboración propia.

Con una confianza del $95 \%$, se puede concluir que existe una diferencia estadísticamente significativa entre Colombia y Ecuador con respecto al grado de conocimiento que se tiene de los recursos. En cuanto a la razón principal por la que utiliza un navegador $u$ otro y sobre los pasos que sigue para realizar una búsqueda en internet, véase tabla 5.

Tabla 5. Diferencia estadística de medias proporcionales de la dimensión de Tecnologías

\begin{tabular}{|c|c|c|c|c|c|}
\hline \multirow[b]{2}{*}{ Dimensión de tecnologías } & \multicolumn{2}{|c|}{ Proporción Pi } & \multirow{2}{*}{$\begin{array}{l}\text { Estadístico } \\
\text { de prueba }\end{array}$} & \multirow{2}{*}{$\begin{array}{l}\text { Valor } \\
\text { crítico } \\
Z \alpha / 2\end{array}$} & \multirow{2}{*}{$\begin{array}{l}\text { Regla de } \\
\text { decisión }\end{array}$} \\
\hline & Colombia & Ecuador & & & \\
\hline $\begin{array}{l}\text { 8. Indica el grado de conocimiento que } \\
\text { tienes de los siguientes recursos. }\end{array}$ & $43,6 \%$ & $37,6 \%$ & 2,348 & 1,96 & $\begin{array}{l}\text { Se rechaza } \\
\text { Ho }\end{array}$ \\
\hline 10. ¿Cuál es el navegador que más utilizas? & $27,3 \%$ & $26,3 \%$ & 0,434 & 1,96 & $\begin{array}{l}\text { Se acepta } \\
\text { Ho }\end{array}$ \\
\hline $\begin{array}{l}\text { 11. Señala la razón principal por la que lo } \\
\text { utilizas. }\end{array}$ & $56 \%$ & $46,1 \%$ & 3,845 & 1,96 & $\begin{array}{l}\text { Se rechaza } \\
\text { Ho }\end{array}$ \\
\hline $\begin{array}{l}\text { 12. ¿Qué pasos sigues para realizar una } \\
\text { búsqueda? }\end{array}$ & $35,9 \%$ & $30,4 \%$ & 2,234 & 1,96 & $\begin{array}{l}\text { Se rechaza } \\
\text { Ho }\end{array}$ \\
\hline
\end{tabular}

Fuente: elaboración propia.

Hay diferencias significativas excepto en la pregunta 10 sobre el navegador que más utiliza.

\section{RECEPCIÓN E INTERACCIÓN}

En Colombia el 34,3\% presenta un grado alto de capacidad para actuar con los diferentes contenidos audiovisuales y en Ecuador el 29,8\% opina que tienen un grado alto de capacidad para actuar con diferentes contenidos audiovisuales (véase tabla 6). 
Tabla 6. Resultados de la dimensión de recepción e interacción. Resultados de presencia de los factores de la dimensión de recepción e interacción

\begin{tabular}{l|l|l|l|l|l|l}
\multicolumn{2}{l|}{ Ecuador } & \multicolumn{3}{l}{ Colombia } \\
\hline Preguntas & Baja & Media & Alta & Baja & Media & Alta \\
\hline $\begin{array}{l}\text { 9. Indica tu grado de capacidad para actuar } \\
\text { con diferentes contenidos audiovisuales. }\end{array}$ & $38 \%$ & $32,2 \%$ & $29,8 \%$ & $36,2 \%$ & $29,5 \%$ & $34,3 \%$ \\
\hline $\begin{array}{l}\text { 17. Valora tu grado de conocimiento sobre } \\
\text { los mecanismos que pueden existir entre los } \\
\text { siguientes medios para presentar quejas o } \\
\text { denuncias sobre contenidos audiovisuales. }\end{array}$ & $32,5 \%$ & $33,9 \%$ & $33,6 \%$ & $36,1 \%$ & $28,1 \%$ & $35,7 \%$ \\
\hline $\begin{array}{l}\text { 18. ¿En qué medida has utilizado los } \\
\text { mecanismos para presentar quejas o } \\
\text { denuncias de los siguientes medios? }\end{array}$ & $62,9 \%$ & $19,4 \%$ & $17,7 \%$ & $72,4 \%$ & $11,7 \%$ & $15,8 \%$ \\
\hline $\begin{array}{l}\text { 19. Indica tu grado de acuerdo respecto a la } \\
\text { conveniencia de denunciar o quejarte. }\end{array}$ & $22,4 \%$ & $29,9 \%$ & $47,6 \%$ & $21,2 \%$ & $21,6 \%$ & $57,2 \%$ \\
\hline $\begin{array}{l}\text { 20. Valora tu grado de usos de los siguientes } \\
\text { mecanismos que utilizas para formular } \\
\text { quejas o denuncias. }\end{array}$ & $41,6 \%$ & $35,7 \%$ & $22,7 \%$ & $49 \%$ & $28,7 \%$ & $22,4 \%$ \\
\hline
\end{tabular}

Fuente: elaboración propia.

En la valoración del grado alto de conocimiento sobre los mecanismos que pueden existir en los medios para presentar quejas o denuncias sobre contenidos audiovisuales los dos países presentan patrones similares: en Colombia el 35,7\% califica de alto grado el conocimiento, y en Ecuador, el 33,6\%. Los dos países presentan similitudes en cuanto al bajo grado de uso de los mecanismos para presentar quejas o denuncias de los medios. Así, se observa que el 72,4\% de los colombianos muestra un bajo grado de uso de los mecanismos frente al 62,9\% de los ecuatorianos. En los dos países se reflejan participaciones sobresalientes en el alto grado respecto a la conveniencia de denunciar o quejarse. El 57,2\% de los colombianos tiene un alto grado de conveniencia de denunciar frente al 47,6\% los ecuatorianos. Se observa que los dos países presentan altas participaciones en la valoración bajo grado en el uso de los mecanismos que se utiliza para formular quejas o denuncias: el 49\% de los colombianos tiene un grado bajo del uso de los mecanismos frente al 41,6\% de los ecuatorianos.

Con una confianza del 95\%, se puede concluir que existe una diferencia estadísticamente significativa entre Colombia y Ecuador con respecto a la conveniencia de denunciar o quejarse (véase tabla 7). 
Tabla 7. Diferencia estadística de medias proporcionales

\begin{tabular}{|c|c|c|c|c|c|}
\hline \multirow[b]{2}{*}{ Dimensión de Recepción e Interacción } & \multicolumn{2}{|c|}{ Proporción Pi } & \multirow{2}{*}{$\begin{array}{l}\text { Estadístico } \\
\text { de prueba }\end{array}$} & \multirow{2}{*}{$\begin{array}{l}\text { Valor } \\
\text { crítico } \\
\mathrm{Z} \alpha / 2\end{array}$} & \multirow{2}{*}{$\begin{array}{l}\text { Regla de } \\
\text { decisión }\end{array}$} \\
\hline & Colombia & Ecuador & & & \\
\hline $\begin{array}{l}\text { 9. Indica tu grado de capacidad para actuar } \\
\text { con diferentes contenidos audiovisuales. }\end{array}$ & $34,3 \%$ & $29,8 \%$ & 1,845 & 1,96 & $\begin{array}{l}\text { Se acepta } \\
\text { Ho }\end{array}$ \\
\hline $\begin{array}{l}\text { 17. Valora tu grado de conocimiento sobre } \\
\text { los mecanismos que pueden existir en los } \\
\text { siguientes medios para presentar quejas o } \\
\text { denuncias sobre contenido audiovisual. }\end{array}$ & $35,7 \%$ & $33 \%$ & 1,092 & 1,96 & $\begin{array}{l}\text { Se acepta } \\
\text { Ho }\end{array}$ \\
\hline $\begin{array}{l}\text { 18. En qué medida has utilizado los } \\
\text { mecanismos para presentar quejas o } \\
\text { denuncias de los siguientes medios. }\end{array}$ & $15,8 \%$ & $17,7 \%$ & 0,994 & 1,96 & $\begin{array}{l}\text { Se acepta } \\
\text { Ho }\end{array}$ \\
\hline $\begin{array}{l}\text { 19. Indica tu grado de acuerdo respecto a } \\
\text { la conveniencia de denunciar o quejarte. }\end{array}$ & $57,2 \%$ & $47,6 \%$ & 3,736 & 2,96 & $\begin{array}{l}\text { Se rechaza } \\
\text { Ho }\end{array}$ \\
\hline $\begin{array}{l}\text { 20. Valora tu grado de uso de los siguientes } \\
\text { mecanismos que utilizas para formular } \\
\text { quejas o denuncias. }\end{array}$ & $22,4 \%$ & $22,7 \%$ & 0,139 & 3,96 & $\begin{array}{l}\text { Se acepta } \\
\text { Ho }\end{array}$ \\
\hline
\end{tabular}

Fuente: elaboración propia.

Respecto a factores de la capacidad para actuar con diferentes contenidos audiovisuales, los conocimientos sobre los mecanismos que pueden existir en los siguientes medios para presentar quejas o denuncias sobre contenidos audiovisuales; en la medida de estos resultados, no existe diferencia significativa estadística con un nivel del 5\%.

\section{Producción y Difusión}

En la elaboración de una historia visualmente bien contada, los dos países presentan comportamientos similares en el grado medio. En Colombia, el 86\% muestra un nivel medio de elaboración de una historia visualmente bien elaborada frente al 75,6\% de los ecuatorianos.

Los dos países presentan grados altos de similitud en ordenar los pasos para realizar un producto audiovisual. Es decir, en Colombia el 86,6\% tiene un alto grado de orden de los pasos a elaborar una historia visualmente bien contada, y en Ecuador, el 80,5\%. 
Tabla 8. Resultados de la dimensión de Producción y Difusión

\begin{tabular}{|c|c|c|c|c|c|c|}
\hline \multirow{2}{*}{$\begin{array}{l}\text { Producción y Difusión } \\
\text { Pregunta }\end{array}$} & \multicolumn{3}{|c|}{ Ecuador } & \multicolumn{3}{|c|}{ Colombia } \\
\hline & Baja & Media & Alta & Baja & Media & Alta \\
\hline $\begin{array}{l}\text { 22. Elabora una historia visualmente bien } \\
\text { contada. }\end{array}$ & $20 \%$ & $75,6 \%$ & $4,2 \%$ & $10,7 \%$ & $86 \%$ & $3,3 \%$ \\
\hline $\begin{array}{l}\text { 23. Ordena los siguientes pasos para realizar } \\
\text { un producto audiovisual. }\end{array}$ & $9,7 \%$ & $9,8 \%$ & $80,5 \%$ & $8,9 \%$ & $4,5 \%$ & $86,6 \%$ \\
\hline
\end{tabular}

Fuente: elaboración propia.

Por lo tanto, con una confianza del $95 \%$, se puede concluir que no existe una diferencia estadísticamente significativa entre Colombia y Ecuador con respecto a la pregunta (22) —elaborar una historia visualmente bien contada- En la pregunta (23) —ordenar los pasos para realizar un producto audiovisual—, existe diferencia significativa estadística con un nivel del 5\%.

Tabla 9. Diferencia estadística de medias proporcionales

\begin{tabular}{|c|c|c|c|c|c|}
\hline Producción y Difusión & \multicolumn{2}{|c|}{ Proporción Pi } & \multirow{2}{*}{$\begin{array}{l}\text { Estadístico } \\
\text { de Prueba }\end{array}$} & \multirow{2}{*}{$\begin{array}{l}\text { Valor } \\
\text { crítico } \\
Z \alpha / 2\end{array}$} & \multirow{2}{*}{$\begin{array}{l}\text { Regla de } \\
\text { decisión }\end{array}$} \\
\hline Pregunta & Colombia & Ecuador & & & \\
\hline $\begin{array}{l}\text { 22. Elabora una historia visualmente bien } \\
\text { contada. }\end{array}$ & $3,3 \%$ & $4,2 \%$ & 0,944 & 1,96 & $\begin{array}{l}\text { Se acepta } \\
\text { Ho }\end{array}$ \\
\hline $\begin{array}{l}\text { 23. Ordena los siguientes pasos para realizar } \\
\text { un producto audiovisual. }\end{array}$ & $86,6 \%$ & $80,5 \%$ & 3,323 & 1,96 & $\begin{array}{l}\text { Se rechaza } \\
\text { Ho }\end{array}$ \\
\hline
\end{tabular}

Fuente: elaboración propia.

\section{IDEOLOGÍAS Y VALORES}

Los dos países presentan participaciones similares en el bajo grado valor de participación en temas sociales y/o políticos: en Colombia el 66,4\% muestra una baja valoración en participar y en el Ecuador, el 64,2\%.

Tabla 10. Resultados de la dimensión de Ideologías y Valores

\begin{tabular}{l|l|l|l|l|l|l} 
Ideología y Valores & \multicolumn{2}{l|}{ Ecuador } & \multicolumn{3}{l}{ Colombia } \\
\hline Pregunta & Baja & Media & Alta & Baja & Media & Alta \\
\hline $\begin{array}{l}\text { 21. Valora tu participación en temas sociales } \\
\text { y/o políticos. }\end{array}$ & $64,2 \%$ & $21,5 \%$ & $14,4 \%$ & $66,4 \%$ & $20 \%$ & $13,7 \%$ \\
\hline
\end{tabular}

Fuente: elaboración propia.

Por lo tanto, con una confianza del $95 \%$, se puede concluir que no existe una diferencia estadísticamente significativa entre Colombia y Ecuador con respecto a valorar la participación en temas sociales y/o políticos. 
Tabla 11. Diferencia estadística de medias proporcionales

\begin{tabular}{|c|c|c|c|c|c|}
\hline Ideologías y Valores & \multicolumn{2}{|c|}{ Proporción Pi } & \multirow{2}{*}{$\begin{array}{l}\text { Estadístico } \\
\text { de Prueba }\end{array}$} & \multirow{2}{*}{$\begin{array}{l}\text { Valor } \\
\text { crítico } \\
\mathrm{Z} \alpha / 2\end{array}$} & \multirow{2}{*}{$\begin{array}{l}\text { Regla de } \\
\text { decisión }\end{array}$} \\
\hline Pregunta & Colombia & Ecuador & & & \\
\hline $\begin{array}{l}\text { 21. Valora tu participación en temas } \\
\text { sociales y/o políticos. }\end{array}$ & $13,7 \%$ & $14,4 \%$ & 0,391 & 1,96 & $\begin{array}{l}\text { Se acepta } \\
\text { Ho }\end{array}$ \\
\hline
\end{tabular}

Fuente: elaboración propia.

\section{EsTÉTICA}

En Colombia, el 35,4\% valora en grado alto los elementos que componen la imagen, en Ecuador, el 38,1\%.

Tabla 12. Resultados de la dimensión de Estética

\begin{tabular}{l|l|l|l|l|l|l} 
Estética & \multicolumn{2}{l}{ Ecuador } & \multicolumn{3}{l}{ Colombia } \\
\hline Pregunta & Baja & Media & Alta & Baja & Media & Alta \\
\hline $\begin{array}{l}\text { 15. Analiza esta imagen desde el punto } \\
\text { de vista estético y valora el grado de } \\
\text { importancia de los siguientes elementos. }\end{array}$ & $22,1 \%$ & $39,8 \%$ & $38,1 \%$ & $27,2 \%$ & $37,4 \%$ & $35,4 \%$ \\
\hline
\end{tabular}

Fuente: elaboración propia.

Con una confianza del 95\%, se puede concluir que no existe una diferencia estadísticamente significativa entre Colombia y Ecuador con respecto a analizar la imagen desde el punto de vista estético.

Tabla 13. Diferencia estadística de medias proporcionales

\begin{tabular}{|c|c|c|c|c|c|}
\hline Estética & \multicolumn{2}{|c|}{ Proporción Pi } & \multirow{2}{*}{$\begin{array}{l}\text { Estadístico } \\
\text { de Prueba }\end{array}$} & \multirow{2}{*}{$\begin{array}{l}\text { Valor } \\
\text { crítico } \\
Z \alpha / 2\end{array}$} & \multirow{2}{*}{$\begin{array}{l}\text { Regla de } \\
\text { decisión }\end{array}$} \\
\hline Pregunta & Colombia & Ecuador & & & \\
\hline $\begin{array}{l}\text { 15. Analiza esta imagen desde el punto } \\
\text { de vista estético y valora el grado de } \\
\text { importancia de los siguientes elementos. }\end{array}$ & $3,3 \%$ & $4,2 \%$ & 0,944 & 1,96 & $\begin{array}{l}\text { Se acepta } \\
\text { Ho }\end{array}$ \\
\hline
\end{tabular}

Fuente: elaboración propia.

\section{DISCUSIÓN Y CONCLUSIONES}

Si bien es cierta la disposición de los jóvenes en Ecuador y Colombia para navegar libremente en la Aldea líquida cuando se tiene la accesibilidad de internet, en los resultados se evidencia la utilización para habilidades más técnicas y operativas que valorativas en cuanto a la competencia mediática. Es el caso llamativo de bajos niveles en la dimensión de Ideología y Valores tanto de Ecuador como de Colombia. Estos resultados al compararlos con estudios similares (Rivera-Rogel et 
al., 2017) revelan comportamientos similares a los de estudiantes de bachillerato españoles (Pérez-Rodríguez et al., 2019).

Gráfico 2. Resultado por dimensiones de Ecuador

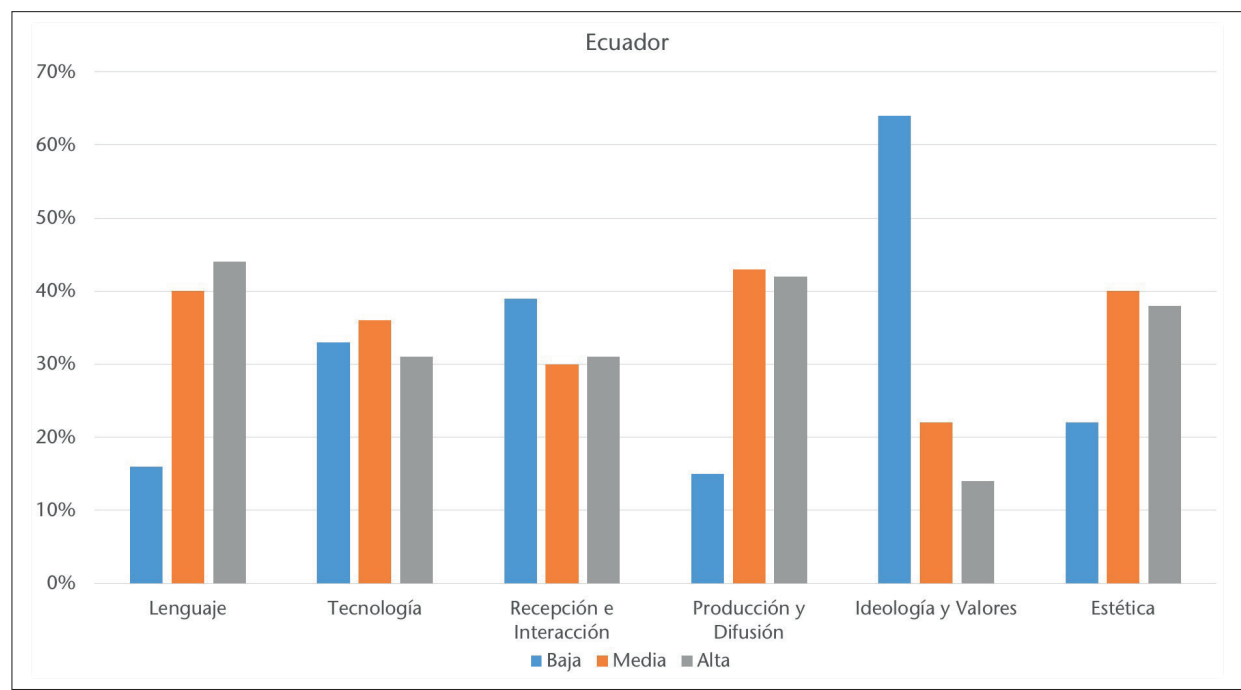

Fuente: elaboración propia.

\section{Gráfico 3. Resultado por dimensiones de Colombia}

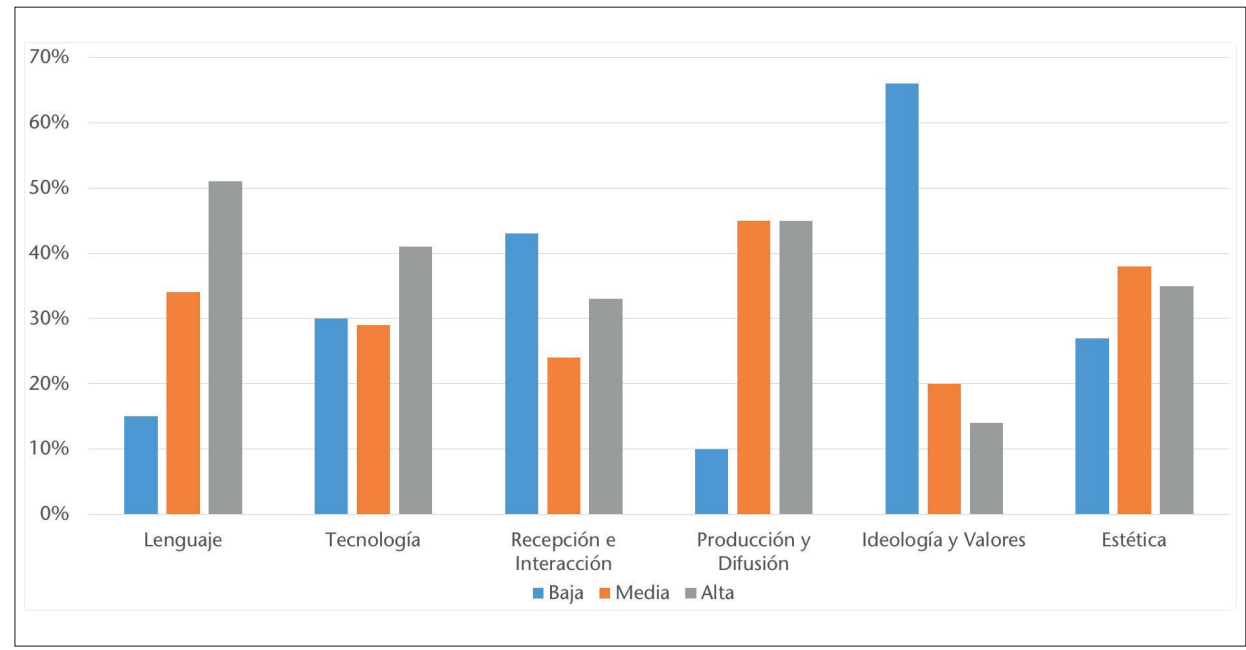

Fuente: elaboración propia. 
En cuanto a la dimensión de Tecnologías, la pregunta (12) “¿Qué pasos sigues para realizar una búsqueda en internet?" revela que, a pesar de que el acceso a internet actualmente en Ecuador se encuentra por encima de Colombia, a la hora de reflejar la capacidad de búsqueda, la habilidad para el conocimiento de esta búsqueda la tienen en mayor medida los jóvenes universitarios de Colombia, lo que resulta significativo. Existen diferencias considerables por países en cuanto al conocimiento de recursos (Pregunta 8) y la razón por la que utilizan un navegador de internet y no otro (Pregunta 11). Pero no existen diferencias en la utilización de navegadores de internet.

Existen diferencias significativas en la dimensión de Lenguaje entre estudiantes ecuatorianos y colombianos, sobre el lenguaje de los medios (Pregunta 13) y los elementos artísticos (Pregunta 16), excepto en la pregunta 14 sobre los diferentes lenguajes en el fragmento de película visualizado.

También existen diferencias significativas en la dimensión de Recepción e Interacción. En cuanto al grado de uso de mecanismos de quejas y denuncias (Pregunta 19), descubrimos que los resultados son significativos entre ambos países. Concretamente, los estudiantes ecuatorianos conocen sus derechos y presentan más quejas y denuncias ante los medios que los estudiantes colombianos.

Se aprecian diferencias significativas entre los estudiantes universitarios colombianos y ecuatorianos en cuanto a la dimensión de Producción y Difusión, en los pasos para realizar un producto audiovisual (Pregunta 23), siendo más alto en los estudiantes colombianos.

Por lo que se refiere a las dimensiones de Estética y de Ideología y Valores no se aprecian resultados significativos entre unos y otros. Así pues, observamos que persiste una brecha relativa a niveles más complejos de alfabetización digital.

\section{Agradecimientos}

Este trabajo se ha elaborado en el marco de Alfamed (Red Interuniversitaria Euroamericana de Investigación en Competencias Mediáticas para la Ciudadanía), con el apoyo del Proyecto I+D+I Coordinado "Competencias mediáticas de la ciudadanía en medios digitales emergentes (smartphones y tablets): prácticas innovadoras y estrategias educomunicativas en contextos múltiples" (EDU201564015-C3-1-R) (MINECO/FEDER), y de la "Red de Educación Mediática" del Programa Español de Investigación Científica-Técnica de Excelencia, Subprograma Estatal de Generación de Conocimiento (EDU2016-81772-REDT), financiados por el Fondo Europeo de Desarrollo Regional (FEDER) y el Ministerio de Economía y Competitividad de España.

Isidro Marín-Gutiérrez (imarin1@utpl.edu. ec). Licenciado en Ciencias Políticas y Sociología por la Universidad de Granada. Doctor en Antropología Social por la Universidad de
Granada. Profesor de la Universidad de Huelva (2004-2013). Desde 2013 es docente e investigador de la Universidad Técnica Particular de Loja (UTPL). Miembro del Consejo Técni- 
co de la revista científica de comunicación y educación Comunicar, del Grupo de Investigación E6, Estudios Sociales e Intervención So-

Diana Rivera-Rogel (derivera@utpl.edu.ec). Profesora titular de Medios Impresos, Titulación de Comunicación Social, Universidad Técnica Particular de Loja desde el 2006. Licenciada en Comunicación Social por la Universidad Técnica Particular de Loja. Doctora en Comunicación y Periodismo por la Univer-

Damian Mendoza-Zambrano (damian. mendoza@uleam.edu.ec). Doctora en Comunicación, línea en educomunicación y competencias mediáticas (Universidad de Huelva). Máster en Comunicación Audiovisual y Educación (Universidad Internacional de Andalucía, Sede Sta. María de La Rábida - UNIA). Especialista en Diseño Curricu-

Ligia Inés Zuluaga-Arias (ligia.zuluagaar@ amigo.edu.co). Colombiana. Comunicadora Social de la UNAD. Especialista en Comunicación Organizacional de la Universidad de Antioquia. Máster en Educación y Desarrollo Humano de la Universidad de Manizales y CINDE. Directora cial (SEJ-216) y del CIM de la Universidad de Huelva, y director del grupo de investigación DocumentaUTPL.

sidad Santiago de Compostela. Directora del Departamento de Ciencias de la Comunicación de la Universidad Técnica Particular de Loja (2012-2014). Fue coeditora internacional de la revista científica Comunicar. Actualmente es directora del Área Sociohumanística de la Universidad Técnica Particular de Loja.

lar Competencia (Chile), diploma en Fotografía-TV (Cuba), licenciada en Relaciones Públicas y Comunicación (UTE Ecuador). Como profesora e investigadora asume la educomunicación desde los nuevos lenguajes de comunicación; guía a los estudiantes para que se empoderen con los medios y las herramientas audiovisuales.

del Programa de Comunicación Social de la Escuela de Comunicación, Publicidad y Diseño de la Universidad Luis Amigó de Medellín. Investigadora en Comunicación-Educación adscrita al grupo de investigación Urbanitas del Programa de Comunicación Social - Funlam.

\section{Bibliografía}

Alcolea-Díaz, G.; Reig, R. y MancinasChávez, R. (2020). "UNESCO's Media and Information Literacy Curriculum for Teachers from the Perspective of Structural Considerations of Information". Comunicar, 28(62), pp. 99-109. DOI: <https://doi.org/10.3916/C622020-09>.

Bravo, D. y Contreras, D. (2000). Encuesta internacional 1997. Alfabetización de la población adulta: desarrollo de competencias y habi- lidades. Santiago de Chile: Universidad de Chile.

Cabero-Almenara, J. y Valencia-Ortiz, R. (2019). "TIC para la inclusión: Una mirada desde Latinoamérica". Aula Abierta, 48(2), pp. 139-146. DOI: <https://doi.org/10.17811/ rifie.48.2.2019.139-146>.

Caldeiro-Pedreira, M. C. y Aguaded-Gómez, I. (2015). “Alfabetización comunicativa y competencia mediática en la sociedad hiper- 
comunicada". Revista Digital de Investigación en Docencia Universitaria (RIDU), 9 (1), pp. 37-55. Disponible en $<\mathrm{http}$ ://cort.as/-IIrK>. Universidad de Chile. Consultado el 28 de febrero de 2020.

Canavos, G. C. (1988). Probabilidad y estadística. México DF: McGraw-Hill.

Clarke, L. W. (2020). "Walk a Day in My Shoes: Cultivating Cross-Cultural Understanding Through Digital Literacy". Reading Teacher, 73(5), pp. 662-665. DOI: <https://doi. org/10.1002/trtr.1890>.

Drotner, K. (2019). “Children's Digital Content Creation: Towards a Processual Understanding of Media Production Among Danish Children". Journal of Children and Media, 14 (2), pp. 221-236. DOI: <https://doi.org/10. 1080/17482798.2019.1701056>.

Ferrés, J. (2007). "La competencia en comunicación audiovisual: Dimensiones e indicadores". Comunicar, 15 (29), pp. 100-107. DOI: <https://doi.org/10.3916/C29-2007-14>.

Ferrés-i Prats, J. y Piscitelli, A. (2012). “Media Competence. Articulated Proposal of Dimensions and Indicators". Comunicar, 19(38), pp. 75-82. DOI: <doi.org/10.3916/C38-201202-08>.

Fontcuberta, J. (1997). El beso de Judas: Fotografía y verdad. Barcelona: Gustavo Gili.

García-Leguizamón, F. (2010). "Educación en medios ayer y hoy: Tópicos, enfoques y horizontes". Magis, Revista Internacional de Investigación en Educación, 2 (4), pp. 279-298. Disponible en <https://goo.gl/oBnj9Z>. Consultado el 20 de febrero de 2020 .

Gozálvez-Pérez, V. y Contreras-Pulido, P. (2014). "Empowering Media Citizenship Through Educommunication". Comunicar, 21(42), pp. 129-136. DOI: <10.3916/c422014-12>.

Gros-Salvat, B. y Contreras, D. (2006). "La alfabetización digital y el desarrollo de competencias ciudadanas". Revista Iberoamericana de Educación (OEI), (42), pp. 103-125. Disponible en <https://goo.gl/1JxdHs>. Consultado el 25 de marzo de 2019.
Guex, P. y Stiefel, F. (2010). "De la souffrance du patient à celle des équipes. Médecine Palliative : Soins de Support - Accompagnement". Éthique, 9(1), pp. 32-35. DOI: $<$ doi:10.1016/j.medpal.2009.12.007 a>.

Hernández-Sampieri, R.; Fernández-Collado, C. y Baptista-Lucio, P. (2014). Metodología de la investigación. México: McGraw-Hill.

Huergo, E. y Trenado, M. (2010). "The Application for and the Awarding of Low-Interest Credits to Finance R\&D Projects". Review of Industrial Organization, 37(3), pp. 237-259. DOI: <doi.org/10.1007/s11151-010-9263-7>.

Izquierdo-Iranzo, P. y Gallardo-Echenique, E. (2020). "Studygrammers: Learning Influencers". Comunicar, 28(62), pp. 111-121. DOI: <https://doi.org/10.3916/C62-2020-10>.

Jitjumnong, K.; Chujai, P. y Kamata, N. (2020). "A Study on Media Literacy of Industrial Education". International Journal of Information and Education Technology, 10(2), pp. 122-126. DOI: $<10.18178 /$ ijiet.2020.10.2.1350 $>$.

Juan, B.; Yasmín, C. y Milton, B. (2020). "Media Competences and Academic Performance of Education Sciences' Teachers of UCE". En: Basantes-Andrade A.; Naranjo-Toro M.; Zambrano-Vizuete, M. y Botto-Tobar M. (eds.). Technology, Sustainability and Educational Innovation (TSIE). TSIE 2019. Advances in Intelligent Systems and Computing. Vol. 1.110. Springer, Cham. DOI: <https://doi. org/10.1007/978-3-030-37221-7_28>.

Martínez, R.; López, R.; Cañedo, M.; Acosta, H.; Granados, J. y Obando F. (2013). "Evolución de la alfabetización digital: Nuevos conceptos y nuevas alfabetizaciones". Medisur, 4 (11), pp. 450-457. Disponible en <http:// medisur.sld.cu/index.php/medisur/article/ view/2467>. Consultado el 5 de abril de 2019.

Mendoza-Zambrano, D. M.; Tirado-Morueta, R. y Marín-Gutiérrez, I. (2017). "Niveles de acceso a internet de los estudiantes del bachillerato en Ecuador". Chasqui. Revista Latinoamericana de Comunicación, (134), pp. 515-529. DOI: <doi.org/10.16921/chasqui. v0i134.2722>. 
Milenkova, V.; Keranova, D. y Peicheva, D. (2020). "Digital Skills and New Media and Information Literacy in the Conditions of Digitization". En: Karwowski, W.; Ahram, T. y Nazir, S. (eds.). Advances in Human Factors in Training, Education, and Learning Sciences. AHFE 2019. Advances in Intelligent Systems and Computing. Vol. 963. Springer, Cham. DOI: <https://doi. org/10.1007/978-3-030-20135-7_6>.

Montoya-Ramírez, N. M. y Zuluaga-Arias, L. I. (2014). "Educar mientras se informa: una propuesta de resignificación de la prensa tradicional". Signo y Pensamiento, 33(64), pp. 3044. DOI: <https://doi.org/10.11144/javeriana. syp33-64.emsi>.

Moreira, M. (2014). "La alfabetización digital y la formación de la ciudadanía del siglo XXI". Integra Educativa, 7(3), pp. 21-33. Disponible en <https://goo.gl/PBw8xQ>. Consultado el 3 de mayo de 2019.

Moreiro, M. A.; Rodríguez, J. y Idáñez, M. J. A. (2010). Materiales y recursos didácticos en contextos comunitarios. Madrid: Grao.

Pérez-Rodríguez, A.; Delgado-Ponce, A.; Marín-Mateos, P. y Romero-Rodríguez, L. M. (2019). "Media Competence in Spanish Secondary School Students. Assessing Instrumental and Critical Thinking Skills in Digital Contexts". Educational Sciences: Theory and Practice, 19(3), pp. 33-48. DOI: <https://doi. org/10.12738/estp.2019.3.003>.

Rivera-Rogel, D.; Zuluaga-Arias, L. I.; Ramírez, N. M. M.; Romero-Rodríguez, L. M. y Aguaded, I. (2017). "Media Competencies for the Citizenship Training of Teachers from Andean America: Colombia and Ecuador". Paidéia (Ribeirão Preto), 27(66), pp. 80-89. DOI: <http:// dx.doi.org/10.1590/1982-43272766201710>.
Sánchez-Carrero, J. y Aguaded-Gómez, J. I. (2013). "El grado de competencia mediática en la ciudadanía andaluza". Estudios sobre el Mensaje Periodístico, 1 (19), pp. 265-280. DOI: $<$ https://doi.org/10.5209/rev_esmp.2013.v19. n1.42521>.

Sánchez-Carrero, J. y Contreras-Pulido, P. (2012). "De cara al prosumidor: producción y consumo empoderando a la ciudadanía 3.0". Revista ICONO14. Revista Científica de Comunicación y Tecnologías Emergentes, 10(3), pp. 62-84. DOI: <https://doi.org/10.7195/ri14. v10i3.210>

Sari, C. M. W.; Huda, I.; Pada, A. U. T.; Rahmatan, H. y Samingan (2020). "Construct Validity of Digital Media Literacy Instrument for Student Teachers". Journal of Physics: Conference Series, 1460 (1), 012053. <DOI: 10.1088/1742-6596/1460/1/012053>.

Scolari, C. A. (2016). "Alfabetismo transmedia: Estrategias de aprendizaje informal y competencias mediáticas en la nueva ecología de la comunicación". Telos: Revista de pensamiento sobre Comunicación, Tecnología y Sociedad. (193), pp. 13-23. Disponible en <http:// hdl.handle.net/10230/27788>. Consultado el 28 de abril de 2019.

Tirado-Morueta, R.; Mendoza-Zambrano, D. M.; Aguaded-Gómez, J. I. y Marín-Gutiérrez, I. (2017). "Empirical Study of a Sequence of Access to Internet use in Ecuador". Telematics and Informatics, 34(4), pp. 171-183. DOI: <https://doi.org/10.1016/j.tele.2016.12.012>.

UNESCO (1986). The Social Relevance of Science and Technology Education. Science and Technology Education Document. París: UNESCO. 
